\title{
Host galaxy properties of quasi-periodically erupting X-ray sources ${ }^{\star}$
}

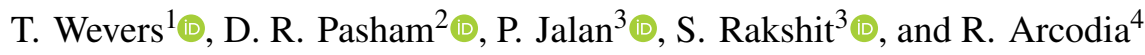 \\ 1 European Southern Observatory, Alonso de Córdova 3107, Casilla 19, Santiago, Chile \\ e-mail: twevers@eso.org \\ 2 MIT Kavli Institute for Astrophysics and Space Research, Cambridge, MA 02139, USA \\ 3 Aryabhatta Research Institute of Observational Sciences, Manora Peak, Nainital 263002, India \\ ${ }^{4}$ Max-Planck-Institut für Extraterrestrische Physik (MPE), Giessenbachstrasse 1, 85748 Garching bei München, Germany
}

Received 17 January 2022 / Accepted 10 February 2022

\section{ABSTRACT}

\begin{abstract}
Context. Quasi-periodic X-ray eruptions (QPEs) are a recently discovered phenomenon, the nature of which remains unclear. Based on their discovery in active galactic nuclei (AGNs), explanations related to an AGN accretion disk or potentially a stellar tidal disruption event (TDE) have been put forward. Alternatives, including highly unequal mass compact object binaries, have also been proposed to explain their properties.

Aims. We perform a systematic study of the five known QPE host galaxies with the aim of providing new insights as to their nature. Methods. We analysed new and archival medium resolution optical spectroscopy of the QPE hosts. We measured emission (and absorption) line fluxes, their ratios, and equivalent widths (EWs) to locate the QPE hosts on diagnostic diagrams. We also measured the velocity dispersion of the stellar absorption lines to estimate their black hole masses.

Results. All QPE host galaxies show emission lines in their optical spectra. Based on their ratios and EWs, we find evidence for the presence of an AGN in all sources, including those previously reported as passive. We measure velocity dispersions between 36 and $90 \mathrm{~km} \mathrm{~s}^{-1}$, implying the presence of low mass $\left(10^{5-6.7} M_{\odot}\right)$ black holes, consistent with literature findings. Finally, we find a significant over-representation (two out of the five sources, or a factor of $13_{-10}^{+13}$ ) of quiescent Balmer strong (post-starburst) galaxies among QPE hosts.

Conclusions. The presence of a narrow line region consistent with an AGN in all QPE host galaxies implies that a long-lived accretion flow likely plays an integral part in the QPE phenomenon. The strong over-representation of quiescent Balmer strong galaxies among QPE hosts can be naturally explained in both the TDE and interacting extreme mass ratio inspiral hypotheses.
\end{abstract}

Key words. accretion, accretion disks - galaxies: Seyfert - galaxies: active - galaxies: nuclei - quasars: supermassive black holes

\section{Introduction}

Quasi-periodic eruptions (QPEs) are recurring X-ray outbursts that have recently been discovered from some extragalactic nuclei (see Miniutti et al. 2019 for the first example). These large amplitude (in excess of an order of magnitude), short (duration of several tens of minutes to hours) eruptions recur on timescales of hours to approximately a day (Miniutti et al. 2019; Giustini et al. 2020; Arcodia et al. 2021). They were first discovered serendipitously in pointed (Miniutti et al. 2019) and archival (Giustini et al. 2020) XMM-Newton observations, and more recently they were also discovered in a blind X-ray survey (Arcodia et al. 2021) performed by the eROSITA instrument (Predehl et al. 2021) aboard the Spectrum-Roentgen-Gamma telescope (Sunyaev et al. 2021) as well as targeted searches in X-ray archives (Chakraborty et al. 2021).

This new class of events contains four known members and one candidate member ${ }^{1}$ to date. Two sources (GSN069 and RXJ1301) were discovered in active galactic nuclei (AGNs).

\footnotetext{
* The host galaxy spectra are only available at the CDS via anonymous ftp to cdsarc.u-strasbg.fr $(130.79 .128 .5)$ or via http: //cdsarc.u-strasbg.fr/viz-bin/cat/J/A+A/659/L2

1 One source is considered a candidate QPE because only a small number (1.5) of QPE-like bursts have been detected so far.
}

The amplitude and duration of QPEs have an energy dependence, being stronger and shorter at higher energies. During quiescence, their X-ray spectrum is consistent with a featureless, cool $(k T \sim 40-60 \mathrm{eV})$ accretion disk model (e.g. Miniutti et al. 2019), and the inferred black hole masses are $\sim 10^{5-6} M_{\odot}$. During the outbursts, a second thermal component (with a higher temperature, $\sim 120 \mathrm{eV}$ ) becomes apparent.

In light of these properties, the first potential explanations that were put forward focussed on instabilities of the inner accretion flow (Sniegowska et al. 2020; Raj \& Nixon 2021). Alternatively, extreme mass ratio inspirals (EMRIs) may also explain the observed QPE properties (King 2020; Suková et al. 2021; Xian et al. 2021; Zhao et al. 2021; Metzger et al. 2022).

Subsequently, two QPEs were discovered in a blind Xray survey (eRO-QPE1 and eRO-QPE2, Arcodia et al. 2021). Finally, the newest candidate member of the QPE family is 2MASXJ0249, which was discovered in the host galaxy of a tidal disruption event (TDE) candidate (Chakraborty et al. 2021). The discovery of QPEs hosted in seemingly passive galaxies (Arcodia et al. 2021) raises some obvious issues for the (pre-existing) AGN accretion disk scenarios, although in the TDE scenario this is not problematic. Furthermore, some properties of recently discovered QPEs (e.g. asymmetries between the fast rise and slower decay phases) cannot be well explained 
Table 1. Details of the spectroscopic observations used in this work and the main results.

\begin{tabular}{|c|c|c|c|c|c|c|c|c|}
\hline Source & $z$ & Telescope/instrument & $\begin{array}{c}\text { Slit width } \\
(\operatorname{arcsec})\end{array}$ & $\begin{array}{r}\sigma_{\text {instr }} \\
\left(\mathrm{km} \mathrm{s}^{-1}\right)\end{array}$ & $\begin{array}{c}\sigma_{\text {meas }} \\
\left(\mathrm{km} \mathrm{s}^{-1}\right)\end{array}$ & $\begin{array}{c}M_{\mathrm{BH}, \sigma} \\
\log _{10}\left(M_{\odot}\right) \\
\end{array}$ & $\begin{array}{c}\mathrm{EW}(\mathrm{H} \alpha) \\
(\AA)\end{array}$ & $\begin{array}{c}\mathrm{H} \delta_{\mathrm{A}} \\
(\AA)\end{array}$ \\
\hline GSN069 & $0.0182^{(a)}$ & Magellan/MagE & 0.7 & 21 & $63 \pm 4$ & $5.99 \pm 0.50$ & $-12.5(4)$ & $3.5 \pm 0.2$ \\
\hline RXJ1301 & $0.0237^{(b)}$ & SDSS & 3 & 70 & $90 \pm 2$ & $6.65 \pm 0.42$ & $-/[-1.7(3)]$ & $6.26 \pm 0.05$ \\
\hline eRO-QPE1 & $0.0505^{(c)}$ & Magellan/MagE & 0.7 & 21 & $56 \pm 5$ & $5.78 \pm 0.55$ & $-2.8(4) /[-3.4(5)]$ & $2.7 \pm 0.3$ \\
\hline eRO-QPE2 & $0.0175^{(c)}$ & Magellan/MagE & 0.7 & 21 & $36 \pm 3$ & $4.96 \pm 0.54$ & $-13.1(2)$ & $1.1 \pm 0.4$ \\
\hline 2MASXJ0249 & $0.0186^{(d)}$ & Keck/ESI & 0.5 & 16 & $43 \pm 4$ & $5.29 \pm 0.55$ & $-33.2(5)$ & $-2.9 \pm 0.9$ \\
\hline
\end{tabular}

Notes. Redshifts are adopted from the literature. For the SDSS spectrum, we report the radius of the fibre, rather than the slit width. The black hole mass is reported using the $M-\sigma$ relation of Gültekin et al. (2009). The EW(H $\alpha)$ lists the measured EW of the line in the rest frame, where values in brackets denote uncertainties as to the last digit. For eRO-QPE1 and RXJ1301, values in square brackets denote measurements on the stellar absorption corrected (i.e. template subtracted) spectra; for RXJ1301, there is no emission before the stellar absorption was corrected. We note that $\mathrm{H} \delta_{\mathrm{A}}$ represents the Lick $\mathrm{H} \delta_{\mathrm{A}}$ absorption index. Negative values indicate lines in emission.

References. ${ }^{(a)}$ Saxton et al. (2011), ${ }^{(b)}$ Dewangan et al. (2000), ${ }^{(c)}$ Arcodia et al. (2021), ${ }^{\left({ }^{d}\right)}$ Esquej et al. (2007).
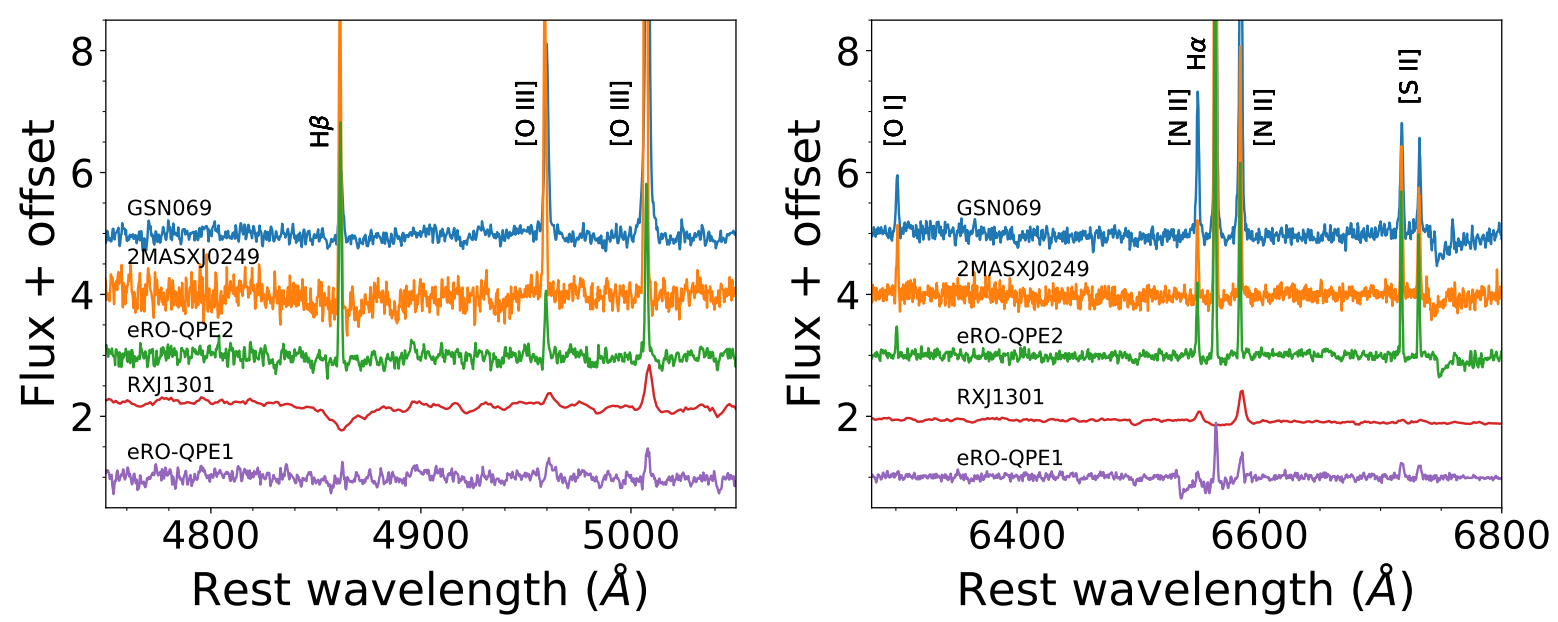

Fig. 1. Insets of the host galaxy spectra of all five QPEs. All spectra show emission lines typical of actively accreting and/or star-forming galaxies, although some emission lines are only visible after subtracting the stellar (galaxy) component.

by accretion disk instabilities, at least according to our current understanding (Arcodia et al. 2021). Alternative hypotheses, which do not require the presence of an AGN accretion disk, have therefore also been proposed. These include extreme mass ratio inspirals of, for example, a white dwarf (WD) orbiting a supermassive black hole (SMBH, King 2020) as well as more generally extreme mass ratio compact object binaries (Arcodia et al. 2021), interacting EMRIs (i.e. multiple objects around a single SMBH, Metzger et al. 2022), and selflensing, massive SMBH binaries (although there are some inconsistencies between amplitudes and timescales in this model, Ingram et al. 2021).

Given that their nature remains unclear, in this Letter we report on the analysis of new and archival optical spectra of the QPE host galaxies. The aim of this work is to systematically characterise their host properties, and ultimately provide new insights as to their nature.

\section{Observations and analysis}

For three QPEs (GSN069, eRO-QPE1, and eRO-QPE2) we present new, medium resolution optical spectroscopy obtained with the Magellan Echelette spectrograph (MagE, Marshall et al. 2008), mounted on the Magellan Baade telescope at Las Campanas Observatory, Chile. The spectra were taken with a $0.7^{\prime \prime}$ slit, leading to a (slit-limited) spectral resolution of $R \approx 5900$, equivalent to an instrumental full width at half maximum (FWHM) broadening of $50 \mathrm{~km} \mathrm{~s}^{-1}$ at $4000 \AA$. These observations were reduced using the dedicated MagE reduction pipeline (Kelson et al. 2000; Kelson 2003). The reduced spectra were subsequently normalised to the continuum by fitting a low order spline function for each order (where emission and absorption lines were excluded through masking and an iterative sigma-clipping routine) and stitched together (see Fig. A.1, black, for an example); inverse-variance mean weighting was used for wavelength regions which are covered by multiple échelle orders.

We also reanalysed an archival Keck/Echelette Spectrograph and Imager (ESI, Sheinis et al. 2002) spectrum (for 2MASXJ0249, presented in Wevers et al. 2019) and an archival Sloan Digital Sky Survey (SDSS) spectrum (for RXJ1301, first presented in Dewangan et al. 2000). Details of all the spectra used in this work can be found in Table 1. Insets of the spectra, focussing on the emission lines used in the analysis, are shown in Fig. 1.

\subsection{Velocity dispersion measurements}

To measure the host velocity dispersion of the stellar absorption lines, we performed full spectrum fitting with the pPXF software (Cappellari 2017), which does a convolution of stellar templates with a variable broadening kernel. We combined pPXF with the ELODIE (Prugniel \& Soubiran 2001; Prugniel et al. 2007) spectral library, which has a slightly 

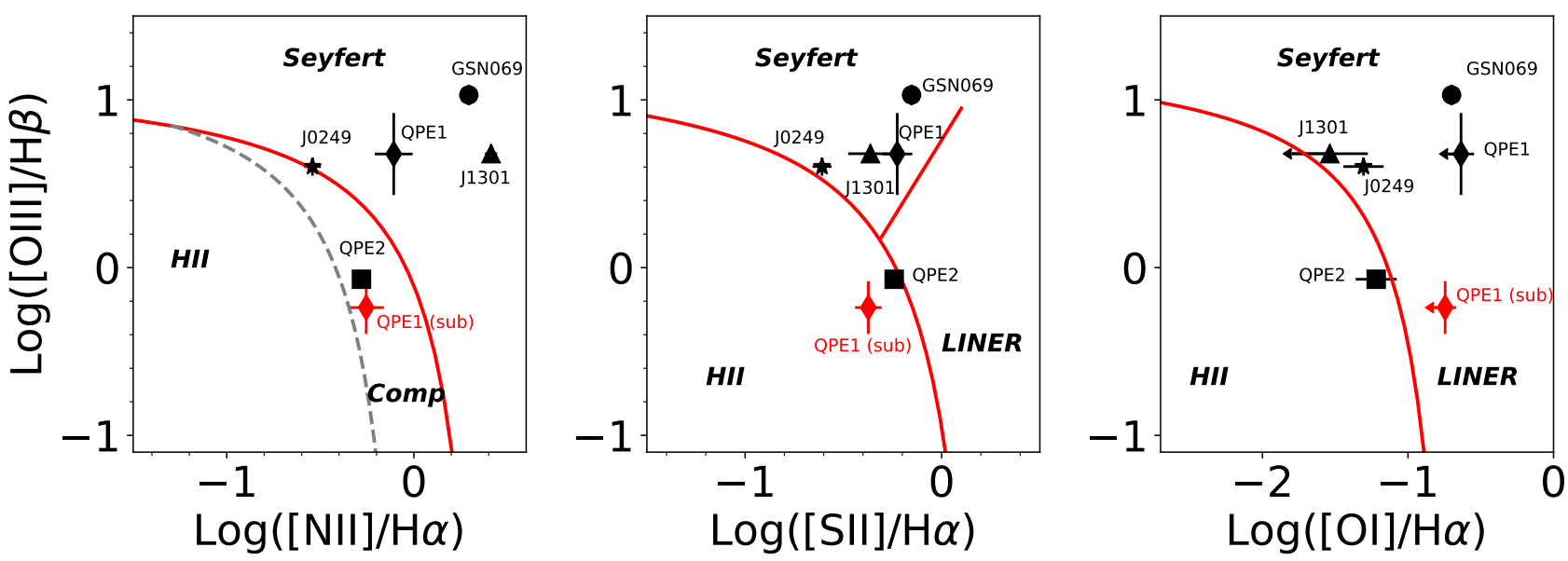

Fig. 2. BPT diagram of the five QPE host galaxies. All sources are beyond the pure star-formation (grey dashed) line, indicating that they very likely host an - in some cases low luminosity - AGN. The data used to reproduce this plot are provided in Table A.1.

better spectral dispersion - $0.54 \AA$ - than the MagE and ESI observations. For the lower resolution SDSS spectrum of RXJ1301, we used the standard MILES single stellar population templates (Falcón-Barroso et al. 2011). The instrumental broadening was subtracted, in quadrature, before measuring the observed line broadening, which is represented by the velocity dispersion $\sigma$, using all available absorption lines in the spectral range 3800-6800 $\mathrm{A}$. We resampled the spectrum 1000 times within the uncertainties and performed a full spectrum fit on each realisation of the data. The reported velocity dispersion and uncertainty are the mean and standard deviation of the resulting distribution, which closely follows a Gaussian distribution. We show an example of the best-fit solution for eRO-QPE1 in Fig. A.1, and the resulting measurements can be found in Table 1.

\subsection{Emission and absorption line measurements}

To measure narrow emission line fluxes, we used the lmfit Python package (Newville et al. 2016) to create a simple model including a polynomial - to account for the continuum flux - and a total of nine Gaussian components, one for each prominent narrow emission line. No broad lines are apparent in the spectra, and no complicated deblending was required. Due to the difficulty in obtaining accurate absolute flux calibrations for échelle spectrographs and since we are primarily interested in their equivalent widths (EWs) and line ratios, we measured the emission line fluxes and EWs on the continuum-normalised spectra. The continuum normalisation is straight-forward in all cases, and, by artificially increasing and decreasing the continuum level, we estimate that this introduces systematic uncertainties of $<25 \%$ in the measurements.

The weakness of $\mathrm{H} \beta$ relative to $\mathrm{H} \alpha$ in eRO-QPE1 and RXJ1301 (for the latter, see Dewangan et al. 2000) indicates that this line is likely affected by stellar absorption. This is typical for post-starburst galaxies, which contain a significant population of A stars, which lead to strong stellar absorption in the Balmer lines. For these two sources, we therefore subtracted the best-fit stellar template obtained with pPXF to account for this effect (see Figs. A.2 through A.5 for visual illustrations), before measuring the narrow emission line fluxes. Lick $\mathrm{H} \delta_{\mathrm{A}}$ absorption indices, optimised for stellar absorption from A stars, were measured as defined in Worthey \& Ottaviani (1997). The main results are presented in Table 1.

\section{Results}

\subsection{The host galaxies of QPEs very likely host an active nucleus}

The host galaxies of each of the five known QPEs show prominent narrow emission lines, although some of these only become apparent after subtracting a template for the stellar component of the galaxy. Using the measured narrow line fluxes, we created diagnostic diagrams to assess the dominant source of ionising photons powering the emission lines. We present the Baldwin-Phillips-Terlevich (BPT) diagram (Baldwin et al. 1981) in Fig. 2, and overlay the classical lines that separate different classes of sources according to the dominant ionisation mechanism (Kauffmann et al. 2003; Kewley et al. 2001): purely star-forming, composite (star-forming + AGN), and AGN (Seyfert/LINER) galaxies. Three out of five sources (2MASXJ0249, GSN069, and RXJ1301) lie within the Seyfert part of the diagrams. eRO-QPE2 is well beyond the extreme starburst line and not affected by any stellar absorption, so it is highly likely that this source contains an AGN (see also Fig. 3). Also, eRO-QPE1 is a more complex case given the strong stellar absorption. The black diamond shows the measurements without correcting for this effect, while the red diamond shows the results from the template subtracted spectrum. In both cases, the source is outside of the pure star-forming region, indicating that it probably also contains an AGN.

In Fig. 3 we show the EW of $\mathrm{H} \alpha$ versus the $[\mathrm{N} \mathrm{II/H} \alpha]$ (WHAN) diagnostic diagram (Cid Fernandes et al. 2011). Here we note that three out of five sources, including eRO-QPE1 and eRO-QPE2, fall within the AGN region. RXJ1301 (a strong poststarburst galaxy) is located among the retired and low ionisation nuclear emission line region (LINER)-like galaxies, which is common for galaxies with relatively weak emission lines (Cid Fernandes et al. 2011). This relative weakness may be due to extinction in the host galaxy as a consequence of its edge-on orientation (Caldwell et al. 1999). There is ample evidence that this source indeed hosts an AGN (e.g. Dewangan et al. 2000; Sun et al. 2013; Giustini et al. 2020). Lastly, 2MASXJ0249 is within the star-formation dominated region of this diagram.

Our medium resolution optical spectroscopy of eRO-QPE1, in particular, significantly differs from the spectrum presented in Arcodia et al. (2021). While no emission lines were clearly visible in their (low resolution) spectrum, we clearly detected emission lines of $\mathrm{H} \beta, \mathrm{H} \alpha$, as well as the typical galaxy 


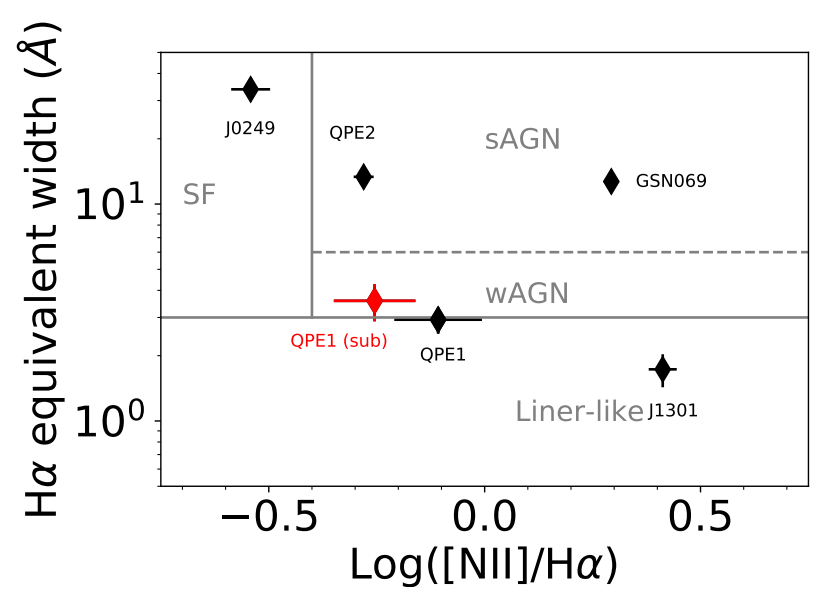

Fig. 3. Diagnostic diagram of the five QPE host galaxies based on $\mathrm{H} \alpha$ EW and relative $[\mathrm{N} \mathrm{II}] / \mathrm{H} \alpha$ strength, based on the selection criteria presented in Cid Fernandes et al. (2011). RXJ1301, a post-starburst galaxy is located among the LINER-like population due to the relative weakness of the $\mathrm{H} \alpha \mathrm{EW}$; whereas in the BPT diagram, it falls firmly in the AGN locus. 2MASXJ0249 is classified as being dominated by star formation, whereas it falls in the Seyfert part of the BPT diagram. The data used to reproduce this plot are provided in Table A.1.

emission lines of [O III], [N II], and [S II]. We speculate that this is due to a combination of higher spectral resolution and smaller slit aperture of our Magellan spectra; the latter leads to less contamination due to non-nuclear galaxy light. The same effects may also be responsible for the different emission line ratios measured for eRO-QPE2, which we find falls within the composite region in the BPT diagram and the AGN region of the WHAN diagram, rather than in the starforming region as reported by Arcodia et al. (2021). Remeasuring the line fluxes in the case of 2MASXJ0249 also led to slightly different results compared to Wevers et al. (2019); we found $\log _{10}(\mathrm{OIII} / \mathrm{H} \beta)=0.60 \pm 0.05$, whereas they reported $\log _{10}(\mathrm{O} \mathrm{III} / \mathrm{H} \beta)=0.4$. We note that Wevers et al. (2019) reported line measurements performed in iraf, which likely have larger systematic errors than the method used in this work.

We conclude from Figs. 2 and 3 that our optical spectroscopy provides strong evidence for an AGN in four out of the five sources. We remark that some well-known discrepancies remain between the BPT and WHAN diagram classification for 2MASXJ0249. In this respect, the WHAN diagram provides the optimal dividing line between SF and AGN dominated line ratios, and as such it is not a strict division (i.e. it is well known that galaxies hosting an AGN can fall to the left this line, and vice versa for star formation dominated galaxies). For the final source (eRO-QPE1), there are more systematic uncertainties, but we deem it probable that it also contains an AGN since the lines cannot be explained by star formation alone. Finally, we note that the narrow line region diagnostics are sensitive to changes in black hole activity only on timescales of several $100-10^{5}$ years (their size in light travel time), and we did not detect broad emission line components in any source. This leaves open the possibility that no AGNs were active in the recent past, and that a different mechanism (e.g. interacting EMRIs, Metzger et al. 2022) is responsible for powering the NLR.

\subsection{The host galaxies of QPEs have low mass black holes}

The velocity dispersion measurements can be used to estimate the black hole mass by exploiting the well-known $M_{\mathrm{BH}}-\sigma$ cor-

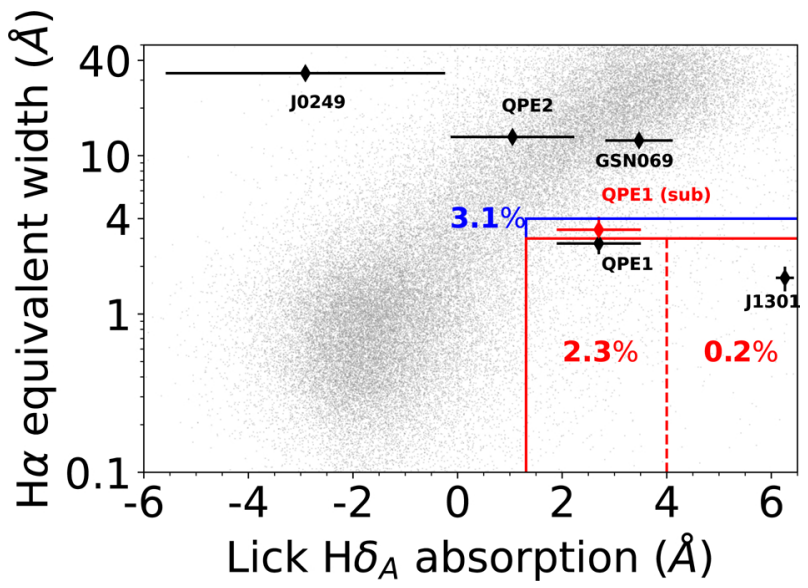

Fig. 4. Spectral properties of SDSS galaxies (grey dots) and QPE hosts (diamonds). Two out of the five QPE hosts lie in a sparsely populated region of this diagram of $\mathrm{H} \alpha \mathrm{EW}$ vs. the Lick $\mathrm{H} \delta_{\mathrm{A}}$ absorption index. The relative fraction of sources within the coloured boxes is shown: $\mathrm{E}+\mathrm{A}$ galaxies represent only $0.2 \%$ of SDSS galaxies (red dashed box), while quiescent Balmer strong galaxies represent 2.3\% (red solid box). The slightly widened (blue) box that encompasses eRO-QPE1 (H $\alpha$ EW $<4 \AA$ ) contains $3.1 \%$ of SDSS galaxies.

relation. The measured velocity dispersions (Table 1) correspond to black hole mass estimates of $10^{5-6} M_{\odot}$ for four out of the five sources, the exception being RXJ1301 for which we inferred $\log _{10}(M)=6.5 \pm 0.4(0.3) M_{\odot}$ using the $M-\sigma$ relation from Gültekin et al. (2009), or $\log _{10}(M)=7.0 \pm 0.3 M_{\odot}$ with the Kormendy \& Ho (2013) relation. Regardless of the specific calibration of the $M-\sigma$ relation that is used, and the associated uncertainties in their calibration at such low values for $\sigma$, these velocity dispersions exclude the presence of massive $\left(>10^{7} M_{\odot}\right)$ black holes, which are typical for the AGN population at large (e.g. Woo \& Urry 2002; Rakshit et al. 2020) for the majority of QPE hosts.

\subsection{Quiescent Balmer strong galaxies are over-represented in QPE hosts}

In Fig. 4 we plotted the QPE hosts among the SDSS galaxy population in terms of $\mathrm{H} \alpha \mathrm{EW}$ (in the rest frame) and the Lick $\mathrm{H} \delta_{\mathrm{A}}$ absorption index ${ }^{2}$ from the MPA-JHU catalogue (Brinchmann et al. 2004). Comparing the location of the QPE hosts to the SDSS galaxy population shows that two sources fall among the blue cloud galaxies with both moderate $\mathrm{H} \alpha$ emission and $\mathrm{H} \delta_{\mathrm{A}}$ absorption. Furthermore, it stands out that two out of the five QPE hosts (eRO-QPE1 and RXJ1301) occupy a very sparsely populated region of the diagram at low $\mathrm{H} \alpha \mathrm{EW}$ and strong $\mathrm{H} \delta_{\mathrm{A}}$ absorption. This region is home to post-starburst galaxies, among which a distinction is made between $\mathrm{E}+\mathrm{A}$ galaxies, which are typically defined as having $\mathrm{H} \alpha \mathrm{EW}<3 \AA$ and a Lick absorption index of $\mathrm{H} \delta_{\mathrm{A}}-\delta_{\mathrm{H} \delta_{\mathrm{A}}}>4 \AA$, and quiescent Balmer strong (QBS) galaxies, which are typically defined as having $\mathrm{H} \alpha \mathrm{EW}<3 \AA$ and a Lick absorption index $>1.31 \AA$. Although, we note that the $\mathrm{H} \alpha \mathrm{EW}$ requirement is somewhat arbitrary. The $\mathrm{H} \alpha$ EW criterion selects galaxies with little ongoing star formation (i.e. well below the star formation main sequence), while the absorption index criterion selects galaxies with a significant population of A stars, which are thought

2 See Appendix B for the SQL queries used to define this sample and the relative fractions reported later. 
to have formed in a recent ( $<1 \mathrm{Gyr})$ starburst. To quantify the rarity of the parameter space where eRO-QPE1 and RXJ1301 are found, we selected SDSS galaxies (from DR17) with $\mathrm{H} \alpha$ $\mathrm{EW}<4 \AA$ and a Lick absorption index $>1.31 \AA$ (i.e. slightly more generous than the typical QBS selection) and found that these represent $3.1 \%$ of the total population ${ }^{3}$. The presence of $2 / 5=40 \%$ of the total QPE sample within this region therefore indicates a significant over-representation, by a factor of $13_{-10}^{+13}$ ( $>2.5$, adopting binomial, small number, $95 \%$ confidence limits, Gehrels 1986), of these galaxies among the QPE hosts compared to SDSS galaxies. The false alarm probability of randomly drawing two sources inside this sparsely populated parameter space is $\sim 0.1 \%$.

\section{Discussion and conclusions}

Black hole masses derived from X-ray spectral fitting as well as estimates based on galaxy scaling relations indicate that the black hole masses of QPE host galaxies are in the range of $10^{5}$-few $\times 10^{6} M_{\odot}$. Our velocity dispersion measurements further suggest that, regardless of the uncertainties in the absolute calibration of the $M-\sigma$ scaling relations at such low measured values, the black hole masses of QPE hosts are $<10^{7} M_{\odot}$, and in some cases they hint at $M_{\mathrm{BH}}$ being as low as $10^{5} M_{\odot}$. While this is atypical for the local galaxy population, it is very similar to the host galaxies of TDEs (Wevers et al. 2017, 2019). The significant over-representation of rare QBS galaxies among QPE hosts (eRO-QPE1 and RXJ1301, Fig. 4) provides a further intriguing link to TDE host galaxies (Arcavi et al. 2014; French et al. 2016). In addition, 2MASXJ0249 and GSN069 have longterm X-ray properties reminiscent of TDEs (Esquej et al. 2007; Auchettl et al. 2017; Miniutti et al. 2019). In summary, four of the five QPEs have an indirect connection - through their longterm X-ray properties or their host galaxy - with the TDE population. Identifying and studying a larger sample of QPEs will reveal whether this is the consequence of small number statistics, selection biases, or whether a true connection between QPEs and TDEs can be established.

One clear difference with the TDE host galaxy population are the emission line ratios, as most TDEs (both UV/optical and $\mathrm{X}$-ray selected) are discovered in passive galaxies without emission lines or in star forming galaxies (e.g. French et al. 2020; Sazonov et al. 2021). Our measurements suggest that the QPE host galaxies contain an ionising source in addition to star formation. The implication is that all QPE host galaxies likely host an AGN. If the association between TDEs and QPEs (based on their host galaxy properties) is real, this may suggest that QPEs are related to special and exotic TDE scenarios, for example as the result of a partial disruption and the subsequent capture of the stellar remnant, which interacts with a pre-existing AGN accretion flow (e.g. Xian et al. 2021). Differences in selection methods between X-ray and UV/optical surveys of TDEs could also contribute to this effect; for example, TDE follow-up observations are more likely to target quiescent galaxies than AGNs.

We note that the QPE quiescent X-ray spectra can be well described by a featureless accretion disk model (Miniutti et al. 2019; Giustini et al. 2020; Arcodia et al. 2021; Chakraborty et al. 2021), which is consistent with the presence of an active nucleus. However, as pointed out in detail by Arcodia et al. (2021), current models that invoke radiation pres-

\footnotetext{
For reference, E+A galaxies make up $\sim 0.2 \%$ of the total population and QBS galaxies with $\mathrm{H} \alpha \mathrm{EW}<3 \AA$ make up $2.3 \%$, see e.g. French et al. (2017).
}

sure instabilities to explain QPE properties do not explain some aspects satisfactorily, including the need for artificially high viscosities or black hole masses which are very strongly incompatible (factor $\sim 1000$ ) with estimates based on galaxy scaling relations presented in this work and the literature.

Alternative hypotheses to explain QPEs include extreme mass-ratio compact object binaries (King 2020; Arcodia et al. 2021). King (2020) argue that a compact object (WD) on a highly eccentric orbit, following a partial stellar disruption and subsequent in-orbit capture of the surviving remnant (i.e. the QPE phenomenon only lasts for approximately several 1000 years), can potentially explain the properties of GSN069. However, Metzger et al. (2022) find that the parameter space for single EMRIs is so small that it is hard to reconcile with the observed QPE occurrence rate, and they argue instead that the interaction between two EMRIs is a more likely scenario.

In the single EMRI scenario, it is not clear why both targeted searches in X-ray archives (e.g. Chakraborty et al. 2021), as well as blind searches (Arcodia et al. 2021) would discover QPEs exclusively around AGNs, which make up only a small minority of galaxies $(\sim 5-10 \%$, e.g. Lopes et al. 2017) in the local Universe. The caveat here is that a sample size of five sources is too small to make conclusive claims at present. We do note that AGN activity is associated with post-starburst evolution, which peaks with a delay of $\sim 250 \mathrm{Myr}$ after the starburst (Wild et al. 2010); Pawlik et al. (2018) estimate an AGN duty cycle of $\sim 50 \%$ during the post-starburst phase. This could indicate that the QPE phase preferentially occurs shortly after the starburst is triggered. The presence of residual emission lines in the two post-starburst QPE hosts could indicate that the bursts occurred recently (Caldwell et al. 1999 found a poststarburst age of $500 \mathrm{Myr}$ for RXJ1301) and that they are still being quenched. Determining the detailed post-starburst ages of a larger sample of QPE hosts could help to elucidate this potential preferential timing and connection.

Metzger et al. (2022) argue that the active phase of an interacting EMRI system is long enough to potentially create its own narrow line region, and furthermore that the presence of a gaseous AGN disk may favour the migration of circular EMRIs into the galactic nucleus. Hence this latter scenario is consistent with our finding that a narrow line region is present in all five known QPEs. Metzger et al. (2022) also argue that post-starburst (or at least, post-merger) galaxies might be favoured QPE hosts due to the presence of migration traps as a result of AGN gas, which can produce a sequence of co-orbiting EMRIs. Finally, the discovery of multiple periodicities in eRO-QPE1 (Pasham et al., in prep.) could be naturally explained in the multiple EMRI scenario.

More generally, at present, the exact link between the narrow emission line diagnostics and the intrinsic ionising source remains ambiguous. The lack of broad emission lines could indicate that none of the SMBHs are currently actively accreting, that is all five QPEs have been discovered in systems with recently shut-down AGN. A different scenario (given the archival X-ray detections of at least two sources, GSN069 and RXJ1301) is that these galaxies host low luminosity AGNs where no luminous broad line region is present, and/or our observations are not sensitive enough to detect the weak broad line region. Finally, if QPEs are a long-lived phenomenon capable of injecting a significant amount of energy into the SMBH surroundings, they could power their own NLR without the need for a traditional AGN (Metzger et al. 2022).

We end by briefly summarising the main results of this work. We have presented a systematic analysis of optical 
spectroscopic observations of the five known QPE host galaxies. This analysis provides new insights regarding the nature of this phenomenon:

1. We have confirmed the low mass nature of the QPE host black holes through velocity dispersion measurements, consistent with the X-ray properties of the QPEs.

2. We have established the presence of narrow emission lines in all QPE host galaxies. Based on their line ratios and EWs, a narrow line region consistent with being (at least partially) ionised by an AGN is present in all QPEs. It remains unclear whether this narrow line region can be powered by QPEs alone, as postulated in some scenarios, or if it requires a longlived AGN.

3. QPE explanations invoking accreting processes (AGNs and TDEs, or a combination of both), as well as interacting EMRI scenarios are compatible with the reported host galaxy properties.

4. We have presented a strong over-representation of rare, quiescent Balmer strong galaxies among the QPE hosts by a factor of $13_{-10}^{+13}$. This provides an intriguing, even if currently unclear, link to the host galaxies of tidal disruption events. The interacting EMRI scenario also provides a natural explanation for this over-representation.

We end by noting that the line ratios presented in this study were measured from ground-based optical spectroscopy. These measurements are known to be affected by finite aperture effects and galaxy light contamination due to limitations imposed by atmospheric seeing, which can distort the conclusions regarding the nature of the ionising continuum. Nevertheless, studying the host galaxy properties of a larger sample of QPEs through groundbased optical spectroscopy may help to further elucidate their nature in the future.

Acknowledgements. We thank the anonymous referee for a prompt and constructive report. This Letter includes data gathered with the $6.5 \mathrm{~m}$ Magellan Telescopes located at Las Campanas Observatory, Chile (P.I. Pasham).

\section{References}

Arcavi, I., Gal-Yam, A., Sullivan, M., et al. 2014, ApJ, 793, 38 Arcodia, R., Merloni, A., Nandra, K., et al. 2021, Nature, 592, 704 Auchettl, K., Guillochon, J., \& Ramirez-Ruiz, E. 2017, ApJ, 838, 149 Baldwin, J. A., Phillips, M. M., \& Terlevich, R. 1981, PASP, 93, 5

Brinchmann, J., Charlot, S., White, S. D. M., et al. 2004, MNRAS, 351, 115

Caldwell, N., Rose, J. A., \& Dendy, K. 1999, AJ, 117, 140

Cappellari, M. 2017, MNRAS, 466, 798

Chakraborty, J., Kara, E., Masterson, M., et al. 2021, ApJ, 921, L40
Cid Fernandes, R., Stasińska, G., Mateus, A., \& Vale Asari, N. 2011, MNRAS, 413, 1687

Dewangan, G. C., Singh, K. P., Mayya, Y. D., \& Anupama, G. C. 2000, MNRAS, 318, 309

Esquej, P., Saxton, R. D., Freyberg, M. J., et al. 2007, A\&A, 462, L49

Falcón-Barroso, J., Sánchez-Blázquez, P., Vazdekis, A., et al. 2011, A\&A, 532, A95

French, K. D., Arcavi, I., \& Zabludoff, A. 2016, ApJ, 818, L21

French, K. D., Arcavi, I., \& Zabludoff, A. 2017, ApJ, 835, 176

French, K. D., Wevers, T., Law-Smith, J., Graur, O., \& Zabludoff, A. I. 2020, Space Sci. Rev., 216, 32

Gehrels, N. 1986, ApJ, 303, 336

Giustini, M., Miniutti, G., \& Saxton, R. D. 2020, A\&A, 636, L2

Gültekin, K., Richstone, D. O., Gebhardt, K., et al. 2009, ApJ, 698, 198

Ingram, A., Motta, S. E., Aigrain, S., \& Karastergiou, A. 2021, MNRAS, 503, 1703

Kauffmann, G., Heckman, T. M., Tremonti, C., et al. 2003, MNRAS, 346, 1055

Kelson, D. D. 2003, PASP, 115, 688

Kelson, D. D., Illingworth, G. D., van Dokkum, P. G., \& Franx, M. 2000, ApJ, 531,159

Kewley, L. J., Dopita, M. A., Sutherland, R. S., Heisler, C. A., \& Trevena, J. 2001, ApJ, 556, 121

King, A. 2020, MNRAS, 493, L120

Kormendy, J., \& Ho, L. C. 2013, ARA\&A, 51, 511

Lopes, P. A. A., Ribeiro, A. L. B., \& Rembold, S. B. 2017, MNRAS, 472, 409

Marshall, J. L., Burles, S., Thompson, I. B., et al. 2008, in Ground-based and Airborne Instrumentation for Astronomy II, eds. I. S. McLean, \& M. M. Casali, SPIE Conf. Ser., 7014, 701454

Metzger, B. D., Stone, N. C., \& Gilbaum, S. 2022, ApJ, 926, 101

Miniutti, G., Saxton, R. D., Giustini, M., et al. 2019, Nature, 573, 381

Newville, M., Stensitzki, T., Allen, D. B., et al. 2016, Astrophysics Source Code Library [record ascl:1606.014]

Pawlik, M. M., Taj Aldeen, L., Wild, V., et al. 2018, MNRAS, 477, 1708

Predehl, P., Andritschke, R., Arefiev, V., et al. 2021, A\&A, 647, A1

Prugniel, P., \& Soubiran, C. 2001, A\&A, 369, 1048

Prugniel, P., Soubiran, C., Koleva, M., \& Le Borgne, D. 2007, VizieR Online Data Catalog: III/251

Raj, A., \& Nixon, C. J. 2021, ApJ, 909, 82

Rakshit, S., Stalin, C. S., \& Kotilainen, J. 2020, ApJS, 249, 17

Saxton, R., Read, A., Esquej, P., Miniutti, G., \& Alvarez, E. 2011, ArXiv e-prints [arXiv:1106.3507]

Sazonov, S., Gilfanov, M., Medvedev, P., et al. 2021, MNRAS, 508, 3820

Sheinis, A. I., Bolte, M., Epps, H. W., et al. 2002, PASP, 114, 851

Sniegowska, M., Czerny, B., Bon, E., \& Bon, N. 2020, A\&A, 641, A167

Suková, P., Zajaček, M., Witzany, V., \& Karas, V. 2021, ApJ, 917, 43

Sun, L., Shu, X., \& Wang, T. 2013, ApJ, 768, 167

Sunyaev, R., Arefiev, V., Babyshkin, V., et al. 2021, A\&A, 656, A132

Wevers, T., van Velzen, S., Jonker, P. G., et al. 2017, MNRAS, 471, 1694

Wevers, T., Stone, N. C., van Velzen, S., et al. 2019, MNRAS, 487, 4136

Wild, V., Heckman, T., \& Charlot, S. 2010, MNRAS, 405, 933

Woo, J.-H., \& Urry, C. M. 2002, ApJ, 579, 530

Worthey, G., \& Ottaviani, D. L. 1997, ApJS, 111, 377

Xian, J., Zhang, F., Dou, L., He, J., \& Shu, X. 2021, ApJ, 921, L32

Zhao, Z. Y., Wang, Y. Y., Zou, Y. C., Wang, F. Y., \& Dai, Z. G. 2021, A\&A, submitted [arXiv:2109.03471] 


\section{Appendix A: Supplementary figures and tables}

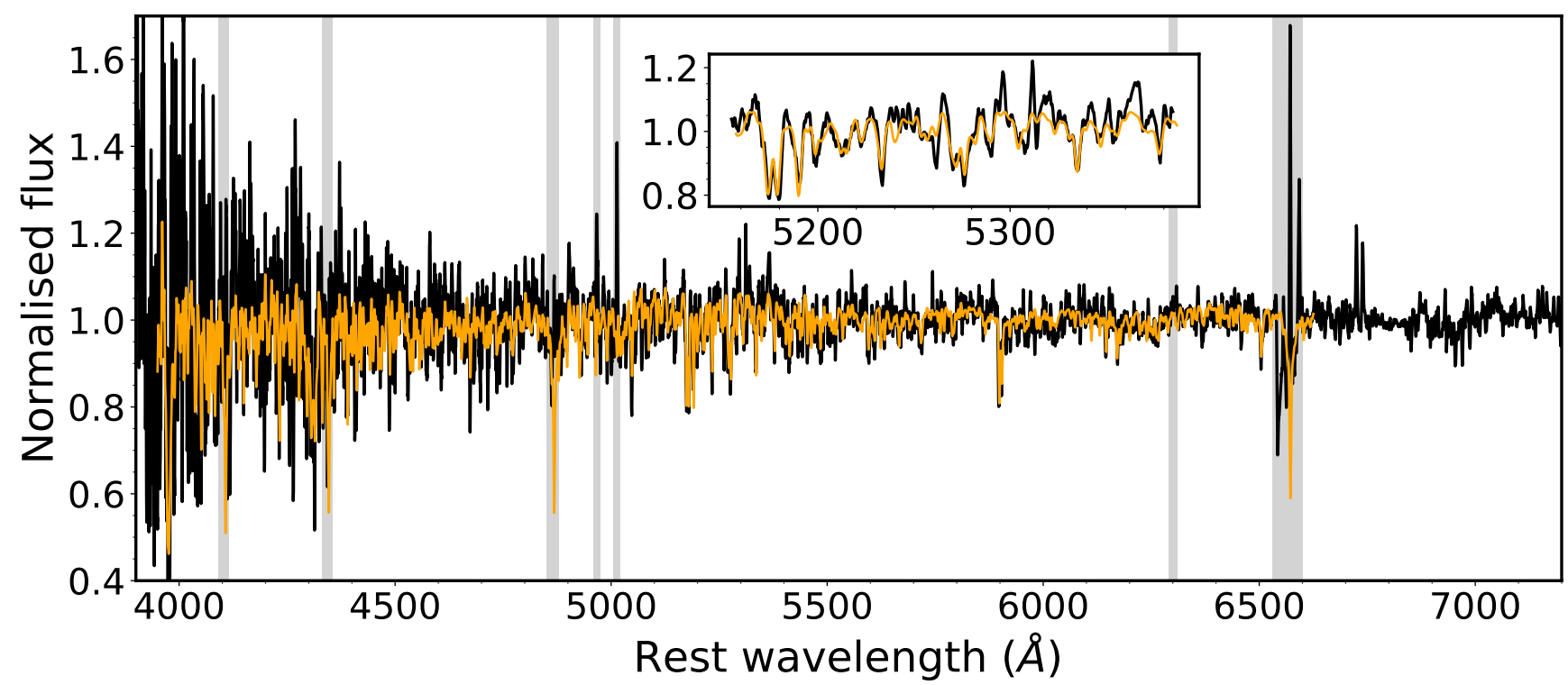

Fig. A.1. Magellan/MagE spectrum (black) of the host galaxy of eRO-QPE1. Overlaid in orange is the best-fit template that was used to measure the host velocity dispersion. The inset shows the region around the $\mathrm{Mg}$ I b triplet to illustrate the quality of the fit. Grey bands indicate host galaxy emission lines, which were excluded from the fit. The spectrum was convolved with a Gaussian kernel with a width of 5 pixels for clarity.

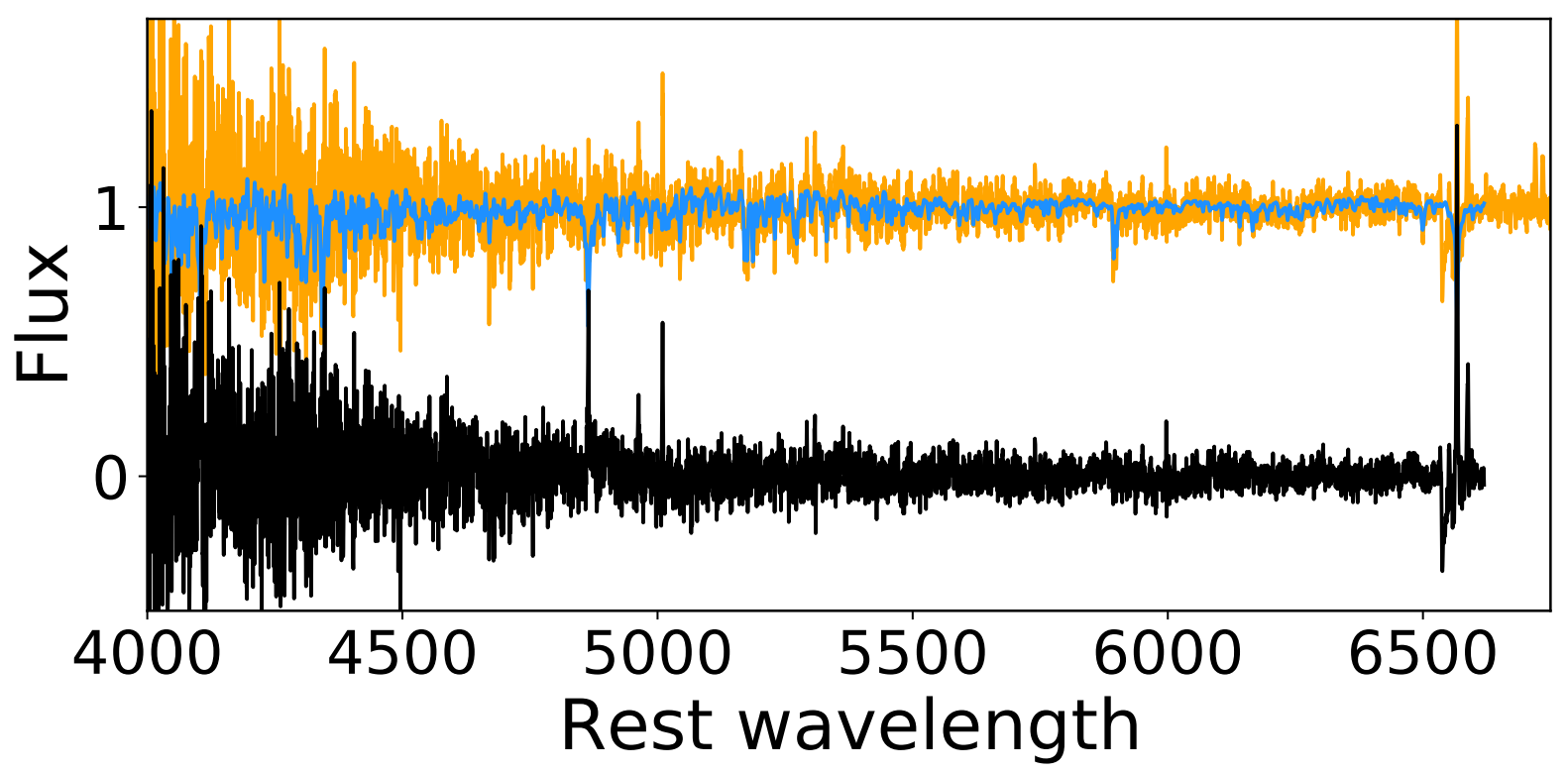

Fig. A.2. Best-fit template (blue) overlaid on the data (orange) and the result of the template subtraction (black) for the host galaxy of eRO-QPE1. 

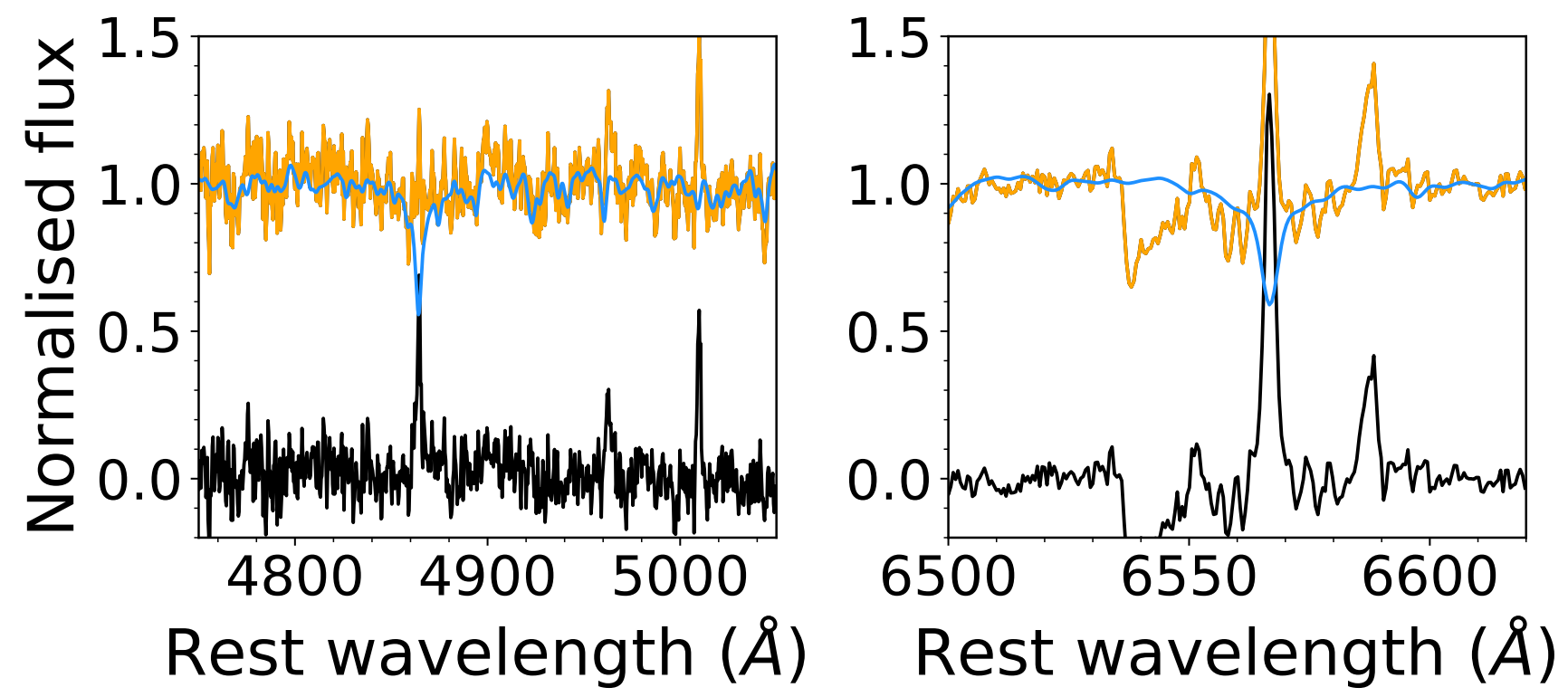

Fig. A.3. Zoom-ins of the $\mathrm{H} \beta$ (left) and $\mathrm{H} \alpha$ (right) regions of the best-fit template (blue) overlaid on the data (orange) and the result of the template subtraction (black) for the host galaxy of eRO-QPE1.

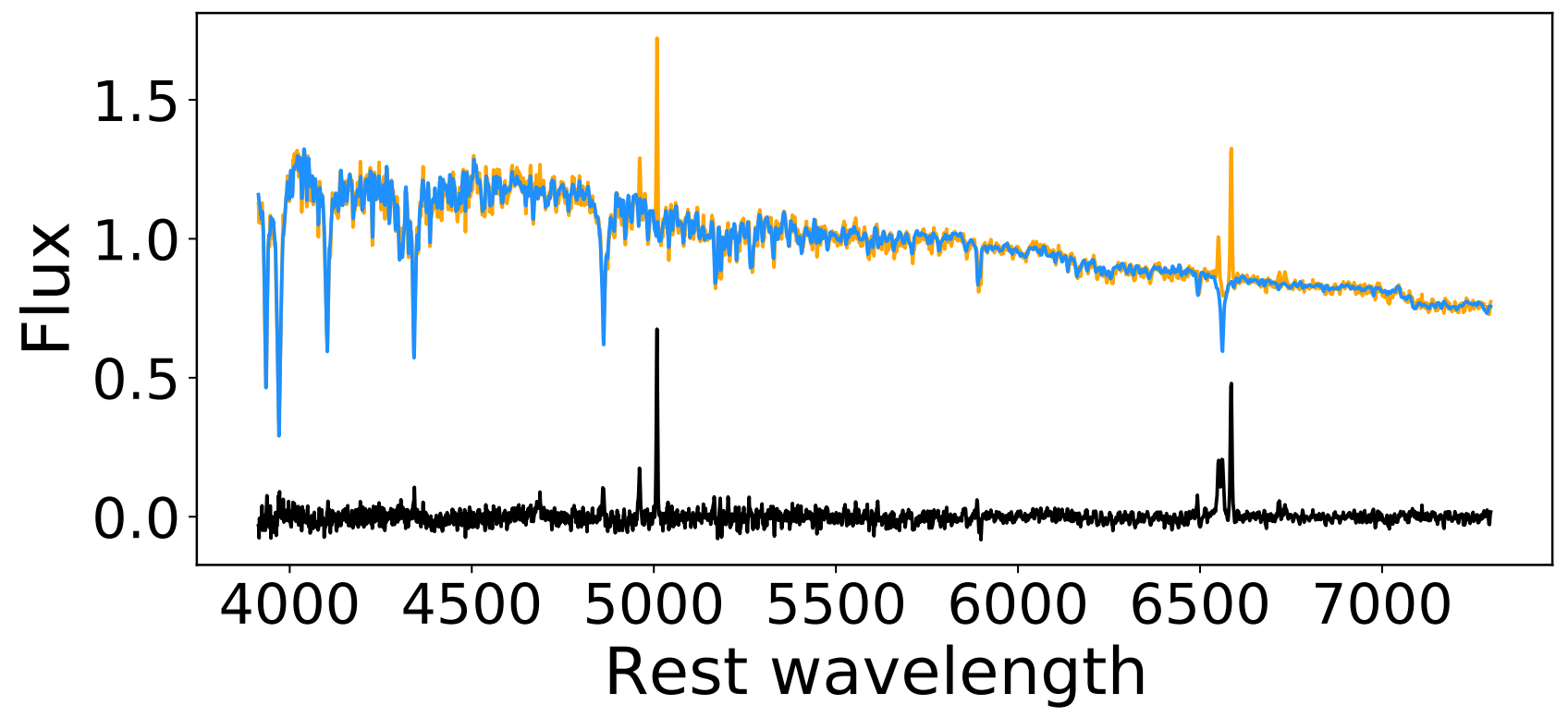

Fig. A.4. Best-fit template (blue) overlaid on the data (orange) and the result of the template subtraction (black) for the host galaxy of RXJ1301. 

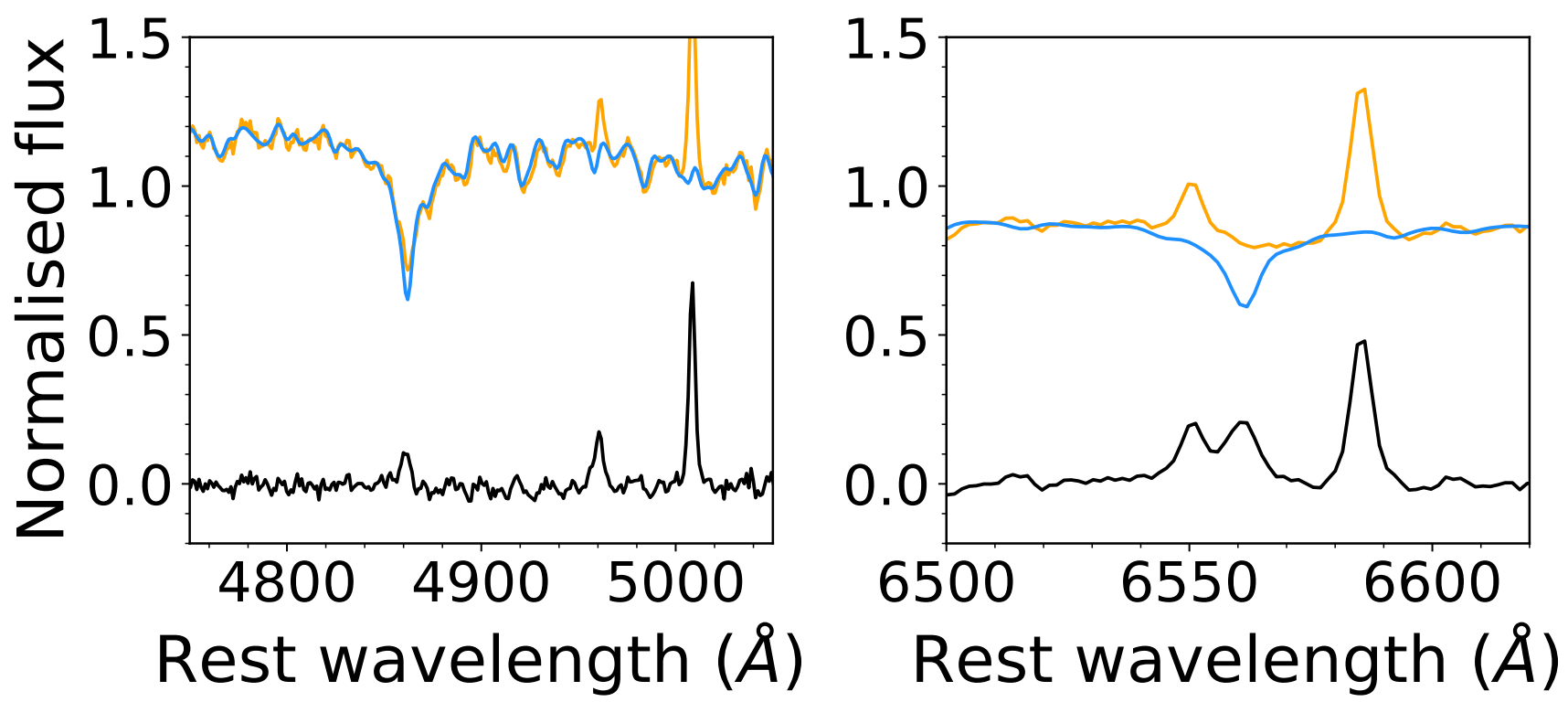

Fig. A.5. Zoom-ins of the $\mathrm{H} \beta$ (left) and $\mathrm{H} \alpha$ (right) regions of the best-fit template (blue) overlaid on the data (orange) and the result of the template subtraction (black) for the host galaxy of RXJ1301.

Table A.1. Line ratios measured from the spectra. For eRO-QPE1 and RXJ1301, values in square brackets denote measurements on the stellar absorption corrected (i.e. template subtracted) spectra; for RXJ1301, there is no emission before the stellar absorption was corrected.

\begin{tabular}{c|cccc}
\hline \hline Source & $\log _{10}(\mathrm{~N}$ II $/ \mathrm{H} \alpha)$ & $\log _{10}(\mathrm{~S} \mathrm{II} / \mathrm{H} \alpha)$ & $\log _{10}(\mathrm{O} \mathrm{I} / \mathrm{H} \alpha)$ & $\log _{10}(\mathrm{O} \mathrm{III} / \mathrm{H} \beta)$ \\
\hline GSN069 & $0.29 \pm 0.02$ & $-0.15 \pm 0.03$ & $-0.70 \pm 0.05$ & $1.03 \pm 0.06$ \\
RXJ1301 & {$[0.41 \pm 0.03]$} & {$[-0.36 \pm 0.11]$} & {$[<-1.5]$} & {$[0.68 \pm 0.05]$} \\
eRO-QPE1 & $-0.11 \pm 0.1[-0.25 \pm 0.1]$ & $-0.23 \pm 0.08[-0.37 \pm 0.07]$ & $<-0.63[<-0.75]$ & $0.68 \pm 0.24[-0.24 \pm 0.16]$ \\
eRO-QPE2 & $-0.28 \pm 0.02$ & $-0.24 \pm 0.02$ & $-1.22 \pm 0.0 .14$ & $-0.07 \pm 0.02$ \\
2MASXJ0249 & $-0.54 \pm 0.05$ & $-0.60 \pm 0.05$ & $<-1.3$ & $0.60 \pm 0.05$ \\
\hline
\end{tabular}




\section{Appendix B: SQL queries}

\section{B.1. SQL query for a clean parent sample}

The following SQL query selects galaxies with a median signal-to-noise ratio of at least ten to discard noisy line measurements. Only galaxies at a redshift $>0.01$ were selected to remove galaxies which are much larger than the SDSS fibre. Unreliable $\mathrm{H} \alpha \mathrm{EW}$ width measurements were excluded by requiring h_alpha_eqw_err $>-1$. This yields a sample of 580182 galaxies.

\section{SELECT}

COUNT $(*)$, s.lick_hd_a, s.lick_hd_a_err,

g.h_alpha_eqw, g.h_alpha_eqw_err

FROM GalSpecLine AS g

JOIN galSpecIndx AS s ON s.specobjid = g.specobjid

JOIN SpecObjAll AS k ON k.specobjid = g.specobjid

WHERE

h_alpha_eqw_err $>-1$

AND k.class = 'GALAXY'

AND k.snMedian $>=10$

AND K.Z $>0.01$

\section{B.2. SQL query for the $E+A$ sample}

This SQL query incorporated the selection criteria for E+A post-starburst galaxies. It yields a sample of 1204 galaxies $(0.2 \%$ of the parent sample).

\section{SELECT}

$\operatorname{COUNT}(*)$

FROM GalSpecLine AS g

JOIN galSpecIndx AS s ON s.specobjid = g.specobjid

JOIN SpecObjAll AS k ON k.specobjid = g.specobjid

WHERE

h_alpha_eqw_err $>-1$

AND h_alpha_eqw $>-3$

AND lick_hd_a - lick_hd_a_err $>4$

AND k.class = 'GALAXY'

AND k.snMedian $>=10$

AND K.Z $>0.01$

\section{B.3. SQL query for the QBS sample}

This SQL query incorporated the selection criteria for QBS galaxies (with h_alpha_eqw > -3) and returned a sample of 14407 galaxies (2.3\% of the parent population). Relaxing the criterion to $\mathrm{h} \_$alpha_eqw $>-4$ to conservatively incorporate QPE1 in this sample results in a sample of 18023 galaxies (3.1\% of the parent sample).

\section{SELECT}

\section{$\operatorname{COUNT}(*)$}

FROM GalSpecLine AS g

JOIN galSpecIndx AS s ON s.specobjid = g.specobjid

JOIN SpecObjAll AS k ON k.specobjid = g.specobjid

WHERE

h_alpha_eqw_err $>-1$

AND h_alpha_eqw $>-3$

AND lick_hd_a > 1.31

AND k.class $=$ 'GALAXY'

AND k.snMedian $>=10$

AND K.Z $>0.01$ 